\title{
Sulforaphane inhibits invasion by phosphorylating ERK1/2 to regulate E-cadherin and CD44v6 in human prostate cancer DU145 cells
}

\author{
XIAOHUI PENG ${ }^{1}$, YAN ZHOU ${ }^{1}$, HUA TIAN $^{1}$, GAOXIANG YANG $^{1}$, \\ CHUNLIU $\mathrm{LI}^{2}$, YANG GENG ${ }^{1}$, SAI WU ${ }^{1}$ and WEI WU ${ }^{1,3}$ \\ ${ }^{1}$ Department of Biochemistry and Molecular Biology, School of Basic Medical Sciences; ${ }^{2}$ Department of Epidemiology and \\ Health Statistics, School of Public Health; ${ }^{3}$ Institute of Brain Tumor, Beijing Institute for Brain Disorders, \\ Capital Medical University, Beijing, P.R. China
}

Received March 9, 2015; Accepted June 11, 2015

DOI: $10.3892 / o r .2015 .4098$

\begin{abstract}
Advanced prostate cancer has highly invasive potential, which may lead to metastasis associated with poor prognosis. Sulforaphane (SFN), abundant in cruciferous vegetables, exhibited effective resistance to carcinogenesis in a variety of tumors. The aim of the present study was to investigate whether SFN inhibited invasion in human prostate cancer cells via sustained activation of ERK1/2 and downstream signaling by an invasion assay, gelatin zymography and western blot analysis. The results showed that SFN inhibited invasion and we characterized the underlying mechanisms in human DU145 prostate cancer cells. SFN $(15 \mu \mathrm{M})$ changed cell morphology leading to short-cell pseudopodia which may suppress tumor migration and invasion. The Transwell assay showed that SFN phosphorylated ERK1/2 in a doseand time-dependent manner and significantly inhibited cell invasion, while the effect was reduced by the ERK1/2 blocker PD98059 $(25 \mu \mathrm{M})$. Furthermore, these effects contributed to the upregulation of E-cadherin and the downregulation of CD44v6 and were eradicated by PD98059. Western blot analysis and gelatin zymography showed that SFN decreased the expression and activity of MMP-2. Thus, SFN inhibited invasion by activating ERK1/2 to upregulate E-cadherin and downregulate CD44v6, thereby reducing MMP-2 expression and activity. E-cadherin is an invasion inhibitor, while CD44v6 and MMP-2 are invasion promoters. Therefore, SFN is a prospective therapeutic agent that may be used to prevent invasion in prostate cancer.
\end{abstract}

Correspondence to: Professor Wei Wu, Department of Biochemistry and Molecular Biology, School of Basic Medical Sciences, Capital Medical University, No. 10, Xitoutiao, You An Men Wai Ave., Feng Tai, Beijing 100069, P.R. China

E-mail: weiwu207@ccmu.edu.cn

Key words: sulforaphane, invasion, prostate cancer, ERK1/2, E-cadherin, CD44v6

\section{Introduction}

Prostate cancer has a high cancer incidence and mortality worldwide (1). Metastasis occurs in over one-third of patients leading to a poor prognosis despite the use of surgery, chemotherapy and radiotherapy (2-4). Thus, it is essential to develop efficient anticancer agents to inhibit metastasis. Invasion, as a key step of metastasis, contributes to cancer progression. The development of anti-invasion drugs is therefore an important strategy to combat prostate cancer.

Dietary intake of cruciferous vegetables was demonstrated to reduce the risks of various types of cancer (5-7). Previous findings have shown that the potential anti-carcinogenic properties of cruciferous vegetables result from sulforaphane (SFN), which is a strong anticancer component (8-11). SFN may suppress cancer progression via a few anti-proliferative mechanisms (12-15). In a previous study, we found that SFN inhibited invasion in human glioblastoma cells through activation of the ERK1/2 signaling pathway (16). However, whether SFN inhibits invasion in human prostate cancer cells remains to be determined. Thus, these effects and the signaling pathways underlying invasion inhibition should be investigated.

Extracellular signal-regulated kinases (ERK1/2) have been shown to have pivotal roles in cell proliferation, differentiation, apoptosis and invasion (17-21). The ERK1/2 pathway triggered oncogene and tumor-suppressor gene expression depending on the time and strength of ERK1/2 activation $(17,21,22)$. It has been indicated that transient ERK1/2 phosphorylation (5-15 min stimulation) resulted in cell proliferation, migration and invasion. By contrast, sustained ( $>15$ min stimulation) activation of ERK1/2 exhibited the opposite effects (17,21-25). Previous results demonstrated that human chorionic gonadotropin $\beta$ (hCG $\beta$ ) promoted cell migration and invasion in human glioblastoma and prostate cancer cells by increasing the expression and activity of MMP-2, resulting from the sustained activation of ERK1/2 (26,27). It was also found that the sustained phosphorylation of ERK1/2 by SFN contributed to the suppression of migration and invasion in human glioblastoma cells (16). Thus, in the present study, we investigated whether SFN 
inhibited invasion in human prostate cancer cells via sustained activation of ERK1/2 and the downstream signaling.

Cadherins, a superfamily of transmembrane glycoproteins, mediate calcium-dependent cell adhesion (28). E-cadherin, a hallmark of epithelial-mesenchymal transition (EMT), belongs to the classic cadherin subfamily and is thought to maintain cell polarity and strengthen intercellular adhesion and acts as a suppressor of invasion. Downregulation of E-cadherin is frequently observed in various metastatic human epithelial cancers (29). It was reported that E-cadherin expression was linked to ERK1/2 activation in tumor cell migration and invasion (30-32). In the present study, we investigated whether E-cadherin was regulated through SFN-induced ERK1/2 phosphorylation in human prostate cancer cells.

The cellular adhesion molecule CD44 is a transmembrane glycoprotein and an EMT marker correlated with breast, prostate, colon, head and pancreatic cancers (33). CD44 isoforms $(\mathrm{CD} 44 \mathrm{v})$ are produced through alternative splicing of the CD44v precursor mRNA (33). Altered expression or dysfunction of CD44 variant 6 (CD44v6) is involved in the aggressive behavior of various types of cancer and correlates with poor prognosis in human malignancies such as breast, leukemia, gastric and colorectal cancer (34-37). Additionally, SFN-induced ERK1/2 phosphorylation contributed to CD44v6 expression in human glioblastoma cells (16). Therefore, we hypothesized that SFN regulates CD44v6 via the activation of ERK1/2 in human prostate cancer cells.

The extracellular matrix (ECM) and basement membranes are crucial barriers against the invasion of malignant cells (38). Matrix metalloproteinases (MMPs) are undoubtedly associated with tumor invasion (39). MMP-2, which is involved in the degradation of type IV collagen and gelatin, is expressed at elevated levels in ovarian, uterine, breast, prostate cancer and melanoma (40-42). Previous results showed that ERK1/2 phosphorylation decreased the expression and activity of MMP-2 in glioblastoma cells (16). Thus, SFN may regulate MMP-2 through the ERK1/2 signaling pathway and contribute to the suppression of invasion in human prostate cancer cells.

In summary, in the present study we investigated the potential of SFN to suppress invasion and identified the underlying molecular mechanisms. Particularly, we aimed to determine key target proteins and establish more powerful strategies to prevent and treat prostate cancer.

\section{Materials and methods}

Reagents. D, L-SFN was purchased from Sigma (St. Louis, MO, USA). RPMI-1640 culture medium was purchased from Hyclone (Logan, UT, USA). Fetal bovine serum (FBS) and penicillin-streptomycin were purchased from Invitrogen-Life Technologies (Carlsbad, CA, USA). Dimethyl sulfoxide (DMSO) was purchased from AppliChem $\mathrm{GmbH}$ (Darmstadt, Germany). An MTS assay kit was purchased from Promega (Madison, WI, USA). Transwell plates used for the invasion assay were purchased from Costar (Corning, Cambridge, MA, USA). Matrigel basement membrane matrix for cell invasion assay was purchased from BD Biosciences (Bedford, MA, USA). PD98059 was purchased from Cell Signaling Technology, Inc. (Shanghai, China). The antibodies,
anti-ERK1/2, anti-phospho-ERK1/2 and anti-E-cadherin were purchased from Cell Signaling Technology. Anti-MMP-2 antibody and anti-CD44v6 were purchased from Abcam (Shanghai, China).

Cell culture. The human DU145 prostate carcinoma cell line was purchased from the Cell Resource Center, Peking Union Medical College (CRC/PUMC). The cells were cultured in RPMI-1640 medium supplemented with 10\% FBS, $100 \mathrm{U} / \mathrm{ml}$ penicillin and $100 \mathrm{U} / \mathrm{ml}$ streptomycin at $37^{\circ} \mathrm{C}$ with $5 \% \mathrm{CO}_{2}$ in a standard humidified incubator. The cells were treated with SFN and ERK1/2 inhibitor PD98059 (25 $\mu \mathrm{M})$ during the logarithmic growth phase for $24 \mathrm{~h}$.

Cell viability assay. DU145 cells were seeded in 96-well plates at $2 \times 10^{3}$ cells/well. After $12 \mathrm{~h}$, the medium in each well was replaced with the medium containing different concentrations of SFN and the plate was incubated for $24 \mathrm{~h}$. Subsequently, the cell viability was detected using an MTS assay kit according to the manufacturer's instructions. The absorbance values were measured by a BioTek ${ }^{\circledR}$ microplate reader (Synergy $^{\mathrm{TM}}$ HT, Winooski, VT, USA) at a wavelength of $490 \mathrm{~nm}$.

Morphological observation. DU145 cells were plated and grown in a 6-well culture plate until they reached $70 \%$ confluence. The cells were then washed with phosphate-buffered saline (PBS) and exposed to different concentrations of SFN $(0,5,10,15,20,25,30,35$ and $40 \mu \mathrm{M})$ for $24 \mathrm{~h}$. Cell morphology was photographed using a digital camera (Olympus, Tokyo, Japan) connected to a phase-contrast microscope (Leica DMIRB, Wetzlar, Germany).

Invasion assay. The Transwell system ( 24 wells, $8 \mu \mathrm{m}$ pore size with polycarbonate membrane) coated with Matrigel was used for the in vitro invasion assays. Matrigel was diluted with serum-free RPMI-1640 to a final concentration of $2 \mathrm{mg} / \mathrm{ml}$. The Transwell inserts were rehydrated with pre-warmed serum-free medium at $37^{\circ} \mathrm{C}$ for $30 \mathrm{~min}$ prior to seeding cells. A total of $2 \times 10^{5}$ cells were suspended in $100 \mu \mathrm{l}$ serum-free medium and were added to the upper chamber. Medium containing $10 \%$ FBS and different concentrations of SFN was then added to the lower chamber, respectively. After $24 \mathrm{~h}$, the cells in the upper chamber were carefully wiped with a cotton swab. The cells invading the lower surface of the filter were fixed with $100 \%$ methanol and stained with crystal violet. The invaded cells on the lower surface of the membrane filter were counted in five randomly fields under microscope, and the cell number was analyzed statistically.

Western blot analysis. DU145 cells from different treatment groups were collected and lysed with RIPA (Thermo Scientific, Waltham, MA, USA) containing protease inhibitors (Roche, Mannheim, Germany). The cell lysate was centrifuged at $12,000 \mathrm{rpm}$ for $20 \mathrm{~min}$. Proteins were separated by SDS-PAGE and transferred onto a nitrocellulose membranes via wet transfer. The membranes were blocked in $1.5 \%$ BSA in TBS Tween-20 (TBS-T) buffer and then incubated with primary antibody at $4^{\circ} \mathrm{C}$ overnight. Subsequently, the membranes were incubated with fluorescence-labeled secondary antibody (LI-COR Bioscience, Lincoln, NE, USA). After being 


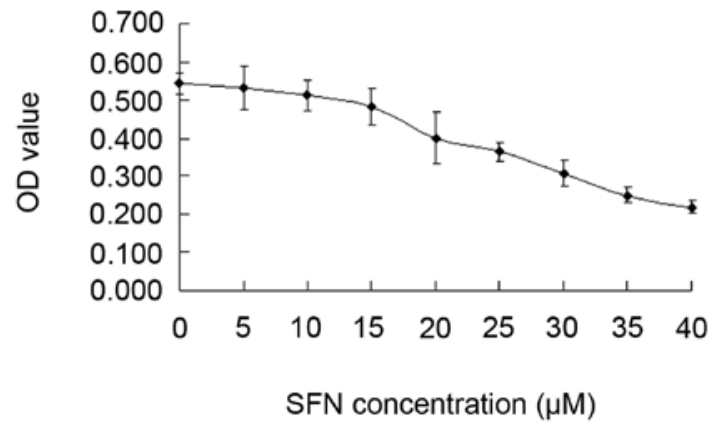

Figure 1. SFN inhibits cell viability. SFN-induced cell viability inhibition was measured by an MTS assay. DU145 cells were incubated in the medium with different concentrations of SFN $(0,5,10,15,20,25,30,35$ and $40 \mu \mathrm{M})$ for $24 \mathrm{~h}$. Cell viability is shown as the percentage of relative absorbance. Data are presented as mean \pm SD from at least three separate experiments. SFN, sulforaphane.

washed with TBS-T for $3 \times 5$ min each, the membranes were scanned using the Odyssey Infrared Imaging System (LI-COR Bioscience).

Gelatin zymography. Following treatment, the medium with MMP-2 activity was collected from an equal number of cells and was centrifuged at 3,000 rpm for $5 \mathrm{~min}$ to remove cell debris. The samples were mixed with non-reduced loading buffer and subjected to a $10 \%$ SDS-polyacrylamide gel containing $0.1 \%$ gelatin as a substrate at $4^{\circ} \mathrm{C}$. After electrophoresis, the gel was rinsed four times with denaturation buffer $(2.5 \%$ Triton $\mathrm{X}-100)$ on a shaker to remove SDS at room temperature and then incubated in developing buffer $(50 \mathrm{mM}$ Tris- $\mathrm{HCl}, \mathrm{pH} 7.5$, $0.2 \mathrm{M} \mathrm{NaCl}, 5 \mathrm{mM} \mathrm{CaCl} \cdot 2 \mathrm{H}_{2} \mathrm{O}$, and $0.02 \%$ Brij-35, $\mathrm{pH} 7.6$ ) for $42 \mathrm{~h}$ at $37^{\circ} \mathrm{C}$. After incubation, the gel was stained using $0.5 \%$ Coomassie Brilliant Blue R-250 for $30 \mathrm{~min}$ and then destained with destaining solution (50\% methanol, $10 \%$ acetic acid, $40 \%$ $\mathrm{ddH}_{2} \mathrm{O}$ ). Enzyme activity was identified as bright bands against the dark blue background of the substrate.

Statistical analysis. Data are presented as means \pm SD. Data were analyzed by ANOVA and subjected to analysis of variance with post-tests for comparison among specific groups. Bonferroni corrections for multiple comparisons against a single group were used. $\mathrm{P}<0.05$ was considered statistically significant. Each experiment was repeated a minimum of three times.

\section{Results}

SFN inhibits cell viability in a dose-dependent manner. DU145 cells were incubated with increasing concentrations of $\operatorname{SFN}(0,5,10,15,20,25,30,35$ and $40 \mu \mathrm{M})$ for $24 \mathrm{~h}$. SFN was dissolved in DMSO. The final concentration of DMSO in the medium was $<0.01 \%$. The medium containing DMSO without SFN was used in the control group. The cell viability was detected using the CellTiter $96^{\circledR}$ AQueous One Solution Cell Proliferation Assay kit (Promega). The results showed that SFN significantly inhibited cell viability in a dose-dependent manner at concentrations of $15-40 \mu \mathrm{M}$ for $24 \mathrm{~h} \quad(\mathrm{P}<0.05$, Fig. 1). Thus, a non-toxic concentration $(<15 \mu \mathrm{M})$ was used as the optimal concentration of SFN for the invasion study.
SFN alters cell morphology in a dose-and time-dependent manner. Untreated DU145 cells presented a morphological change with some cell pseudopodia. We found that SFN-treated cells exhibited less pseudopodia and became rounded with the doses and times (Fig. 2). Pseudopodia are required in the migration and invasion of tumor cells. Therefore, we suggested that SFN may inhibit invasion in DU145 cells.

SFN inhibits cell invasion in a dose-dependent manner. Matrigel-coated Transwells were used to determine the effect of SFN on cell invasion. DU145 cells were treated with different concentrations of $\operatorname{SFN}(0,5,10$ and $15 \mu \mathrm{M})$. The results showed that SFN significantly reduced cell invasion vs. the control group in DU145 cells (Fig. 3A). In order to determine whether SFN inhibited cell invasion by activating ERK1/2, we continued to treat cells with or without PD98059, the blocker of ERK1/2. The invasive ability of the cells treated with PD98059 and SFN was improved vs. the SFN-only treatment cells (Fig. 3B).

SFN activates ERK1/2 in a dose- and time-dependent manner. The ERK1/2 phosphorylation was tested in SFN-treated DU145 cells. SFN was added to the medium at different concentrations $(0,5,10$ and $15 \mu \mathrm{M})$ for $24 \mathrm{~h}$. Western blot analysis showed that SFN induced ERK1/2 phosphorylation in a dose-dependent manner (Fig. 4A). We treated the cells with $15 \mu \mathrm{M} \mathrm{SFN}$ at different time-points $(0,12,24$ and $36 \mathrm{~h})$. Western blot analysis revealed that ERK1/2 phosphorylation followed a time-dependent manner and increased to the peak at $24 \mathrm{~h}$ (Fig. 4B). PD98059 significantly diminished ERK1/2 phosphorylation and the level of activation was higher than that of the control ( $0 \mu \mathrm{M}$ of SFN) (Fig. 4C).

SFN upregulates E-cadherin by activating ERK1/2. In the present study, we investigated whether SFN regulated E-cadherin in DU145 cells. Western blot analysis showed that SFN upregulated E-cadherin significantly in a dose-dependent manner (Fig. 5A). To clarify whether the increased level of E-cadherin by SFN treatment was caused by phosphorylation of ERK1/2, we pretreated cells with PD98059. Western blot analysis showed that PD98059 significantly abolished the upregulation of E-cadherin triggered by SFN (Fig. 5B). This result indicated that $S F N$ upregulated $E$-cadherin via sustained ERK1/2 phosphorylation.

SFN downregulates CD44v6 in a sustained manner. Western blot analysis showed that SFN downregulated CD44v6 significantly in a dose-dependent manner (Fig. 6A). The cells were treated with PD98059 to verify whether SFN regulated CD44v6 via ERK1/2 phosphorylation. However, western blot analysis showed that PD98059 was able to reverse the reduction of CD44v6 triggered by SFN (Fig. 6B). This result indicated that SFN markedly downregulated CD44v6 by phosphorylating ERK1/2.

SFN downregulates pro-MMP-2 (72 kDa) expression and decreases active MMP-2 (64 kDa) activity. Western blot analysis showed that SFN downregulated pro-MMP-2 expression (Fig. 7A). Gelatin zymography revealed that SFN reduced active MMP-2 in a dose-dependent manner (Fig. 7C). 
$\operatorname{SFN}(\mu \mathrm{mol} / \mathrm{l})$

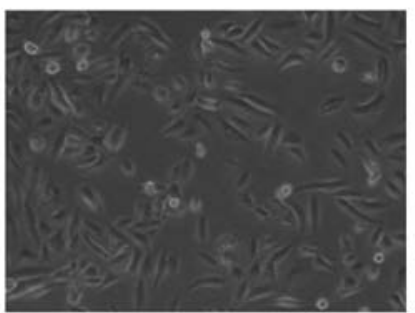

0

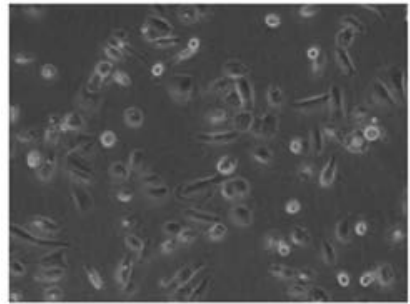

15

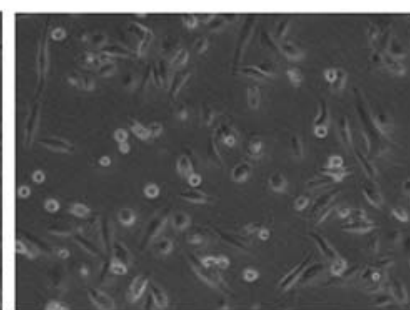

5

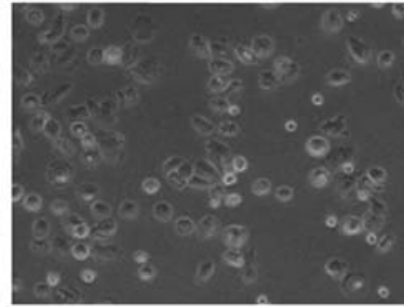

20

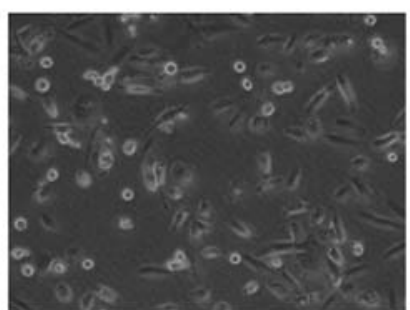

10

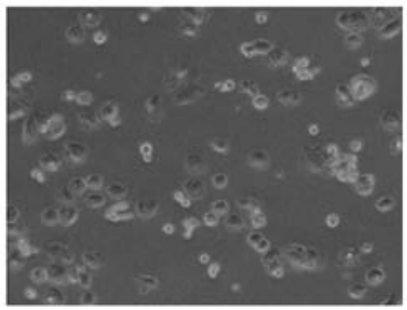

25

Figure 2. SFN alters cell morphology. Cell shapes were altered when the cells were treated with increasing concentrations of SFN ( $0,5,10,15,20$ and $25 \mu \mathrm{M})$ for $24 \mathrm{~h}$. Morphological observation was recorded by a Leica DMIRB microscope. Magnification, x100. SFN, sulforaphane.

A

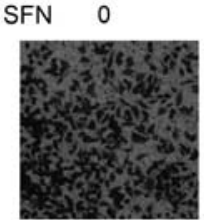

10
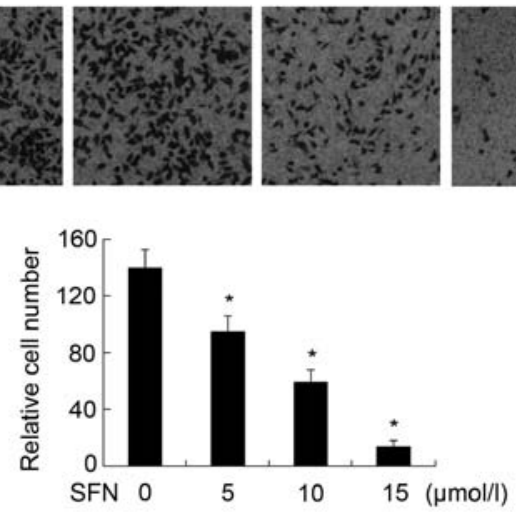

B
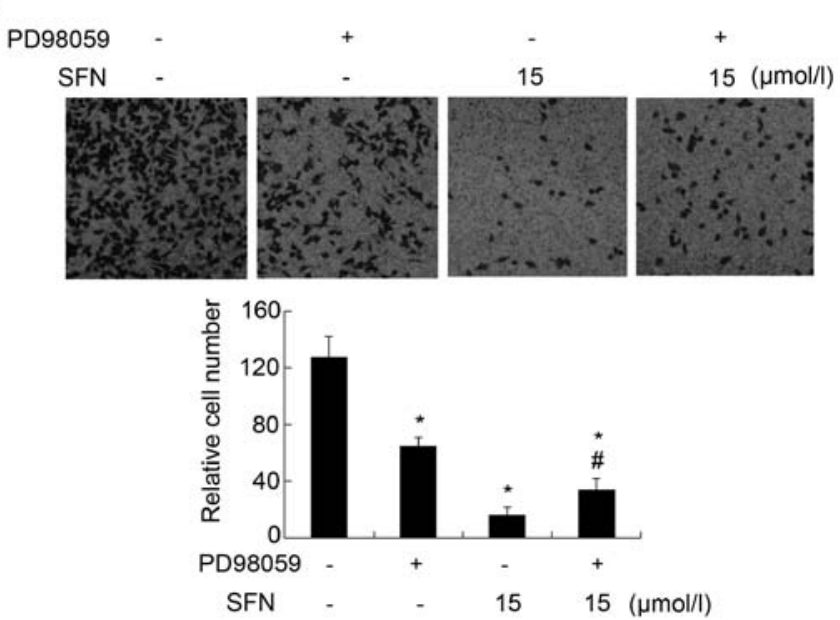

Figure 3. SFN inhibits cell invasion. (A) Cell invasion was assessed by a Matrigel-coated Transwell system. We seeded $2 \times 10^{5}$ cells in the medium with increasing concentration of SFN $(0,5,10$ and $15 \mu \mathrm{M})$ for $24 \mathrm{~h}$. (B) Under the same conditions we incubated the cells with SFN $(15 \mu \mathrm{M})$ with or without PD98059 to detect the cell invasive ability. ${ }^{*}$, indicates $\mathrm{P}<0.05$ vs. control group. ", indicates $\mathrm{P}<0.05$ vs. SFN-only group. Data are shown as means \pm SD from three separate experiments. SFN, sulforaphane.
Furthermore, the cells were pretreated with PD98059 to verify whether this regulation of MMP-2 resulted from the phosphorylation of ERK1/2. No obvious variance between SFN-only treatment and PD98059 plus SFN treatment was identified, suggesting that blocking the phosphorylation of ERK1/2 did not eradicate inhibition of MMP-2 expression and activation induced by SFN. Thus, the ERK1/2 signaling pathway was dispensable in the SFN-mediated reduction of MMP-2 expression and activation thereof. (Fig. 7B and D).

\section{Discussion}

SFN has been identified as a promising agent in preclinical evaluation and is able to block carcinogenesis effectively. Previous studies have reported that normal human bronchial epithelial and normal prostate epithelial cells are more resistant to SFN-induced apoptosis compared with human lung and prostate cancer cells (9). Although SFN potentially resists metastasis by suppressing cell migration and invasion in some types of cancer, the involved mechanisms remain to be determined (16,43-45).

In the present study, we show that SFN potently inhibited proliferation and invasion in vitro. The results of the MTS assay showed that SFN suppressed proliferation significantly at a concentration of $>15 \mu \mathrm{M}$. The morphological observation showed that cell pseudopodia were markedly different. These results provide an initial reference to determine the optimal working concentration in invasion study.

The ERK1/2 signaling pathway contributes to tumor occurrence, development and metastasis $(17,21,22)$. Our results indicated that SFN induced ERK1/2 phosphorylation in a dose- and time-dependent manner. We also found that SFN suppressed invasion through the activation of ERK1/2. These data are consistent with those obtained in a previous study (16). One possibility for this activation of ERK1/2 may result from 

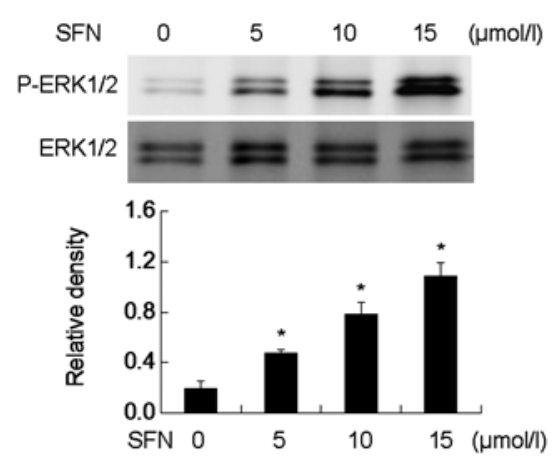

B
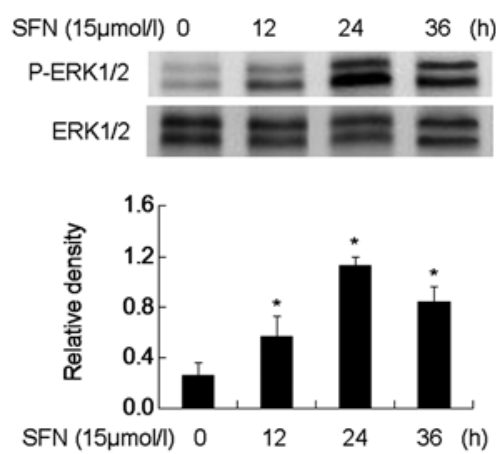

$\mathrm{C}$
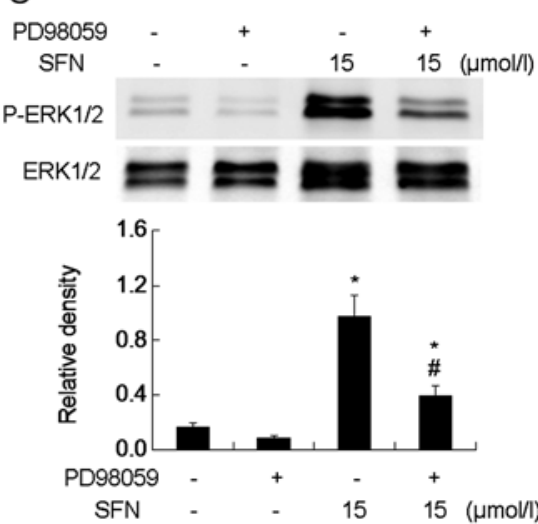

Figure 4. Western blot analysis shows that SFN activated ERK1/2. (A) We treated the DU145 cells in the medium with different concentrations of SFN (0, 5,10 and $15 \mu \mathrm{M}$ ) for $24 \mathrm{~h}$. (B) We treated the DU145 cells with $15 \mu \mathrm{M}$ SFN for $0,12,24$ and $36 \mathrm{~h}$. (C) Prior to adding SFN, we pretreated DU145 cells with PD 98059 for $1 \mathrm{~h}$, then incubated cells for $24 \mathrm{~h} .{ }^{*}$, indicates $\mathrm{P}<0.05$ vs. control group. ${ }^{*}$, indicates $\mathrm{P}<0.05$ vs. SFN-only group. Data were shown as means \pm SD from three separate experiments. SFN, sulforaphane.
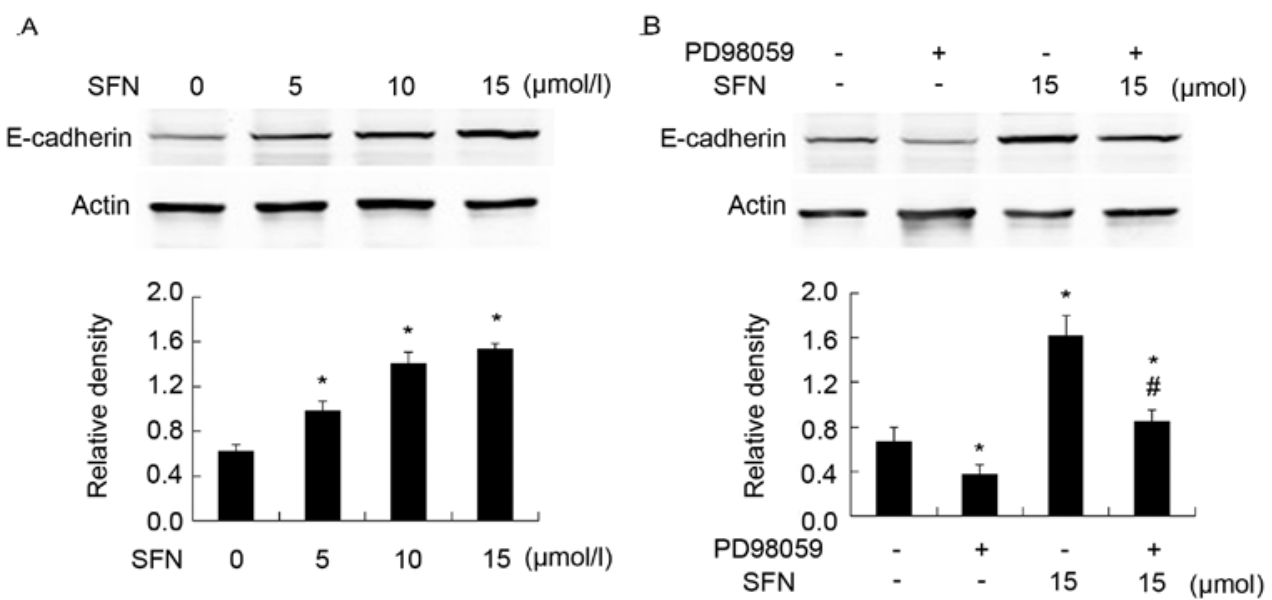

Figure 5. Western blot analysis shows that SFN upregulated E-cadherin by activating ERK1/2. (A) We exposed the cells to different concentrations of SFN (0, $5,10$ and $15 \mu \mathrm{M})$ for $24 \mathrm{~h}$. (B) We treated the cells with PD98059 only, SFN $(15 \mu \mathrm{M})$ only and both PD98059 and SFN (15 $\mu \mathrm{M})$ for $24 \mathrm{~h}$. *, indicates P<0.05 vs. control group. ${ }^{\#}$, indicates $\mathrm{P}<0.05$ vs. SFN-only group. Data are shown as means \pm SD from three separate experiments. SFN, sulforaphane; ERK1/2, extracellular signal-regulated kinases.

A
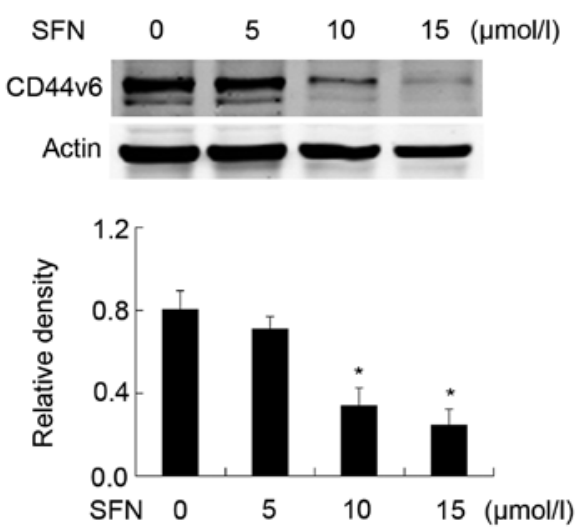

B

PD98059
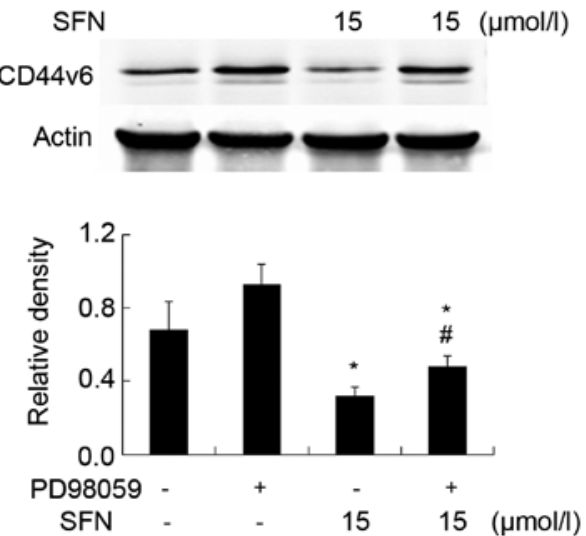

Figure 6. Western blot analysis shows that SFN downregulated CD44v6 by activating ERK1/2. (A) We exposed the cells to different concentrations of SFN $(0,5,10$ and $15 \mu \mathrm{M})$ for $24 \mathrm{~h}$. (B) We treated the cells with PD98059 only, SFN $(15 \mu \mathrm{M})$ only and PD98059 plus SFN $(15 \mu \mathrm{M})$ for $24 \mathrm{~h}$. ${ }^{*}$, indicates P<0.05 vs. control group. ", indicates $\mathrm{P}<0.05$ vs. SFN-only group. Data are shown as means \pm SD from three separate experiments. SFN, sulforaphane; ERK1/2, extracellular signal-regulated kinases; CD44v6, CD44 variant 6. 
A
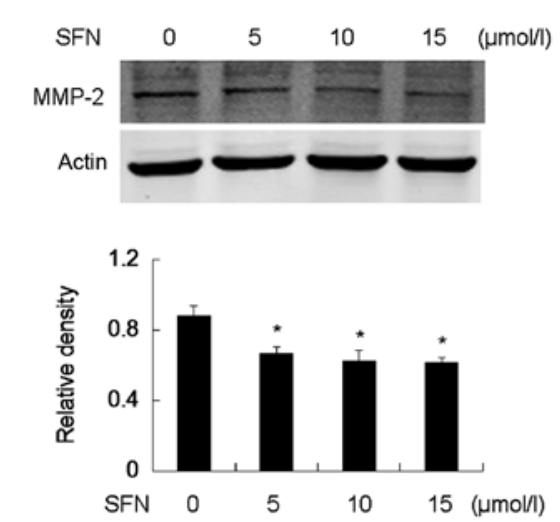

C

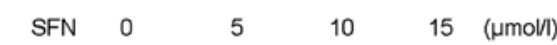

MMP-2
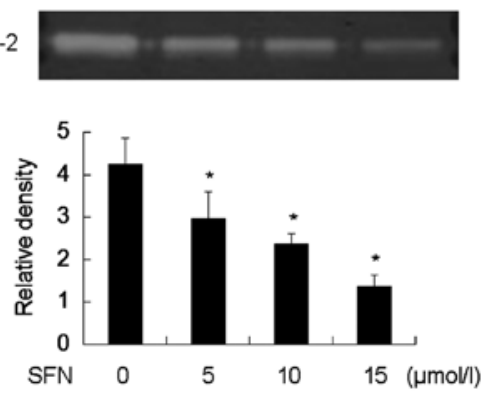

B
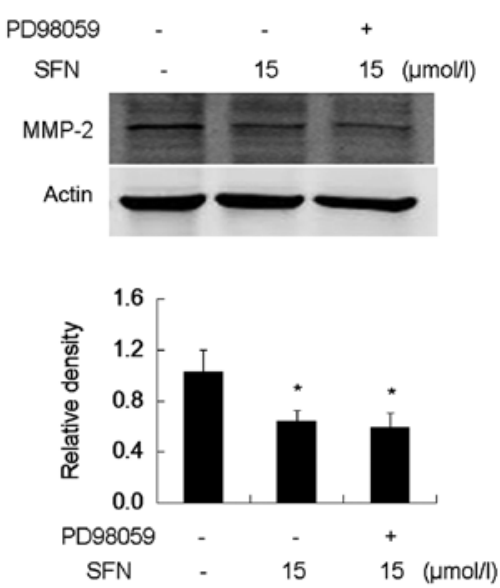

D

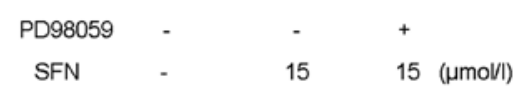

MMP-2
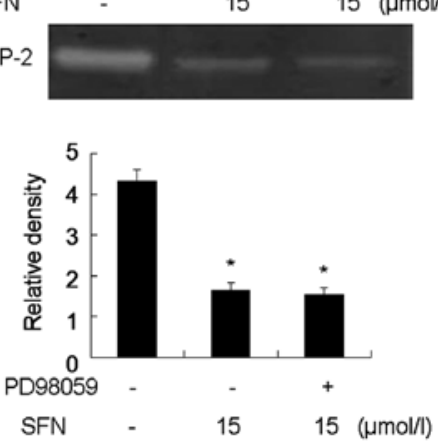

Figure 7. SFN downregulates pro-MMP-2 expression and decreases active-MMP-2 activity. (A) We treated the cells in the medium with different concentrations of SFN $(0,5,10$ and $15 \mu \mathrm{M})$ for $24 \mathrm{~h}$. Western blot analysis indicated that SFN downregulated pro-MMP-2 in a dose-dependent manner. (B) We treated the cells with SFN $(15 \mu \mathrm{M})$ only and both PD98059 and SFN $(15 \mu \mathrm{M})$ for $24 \mathrm{~h}$. (C) The conditioned medium from the above treatment for gelatin zymography assay was collected. (D) We incubated the cells in serum-free medium with SFN $(15 \mu \mathrm{M})$ adding or not adding PD98059 for $24 \mathrm{~h}$. ", indicates P<0.05 vs. control group. Data are shown as means \pm SD from three separate experiments. SFN, sulforaphane.

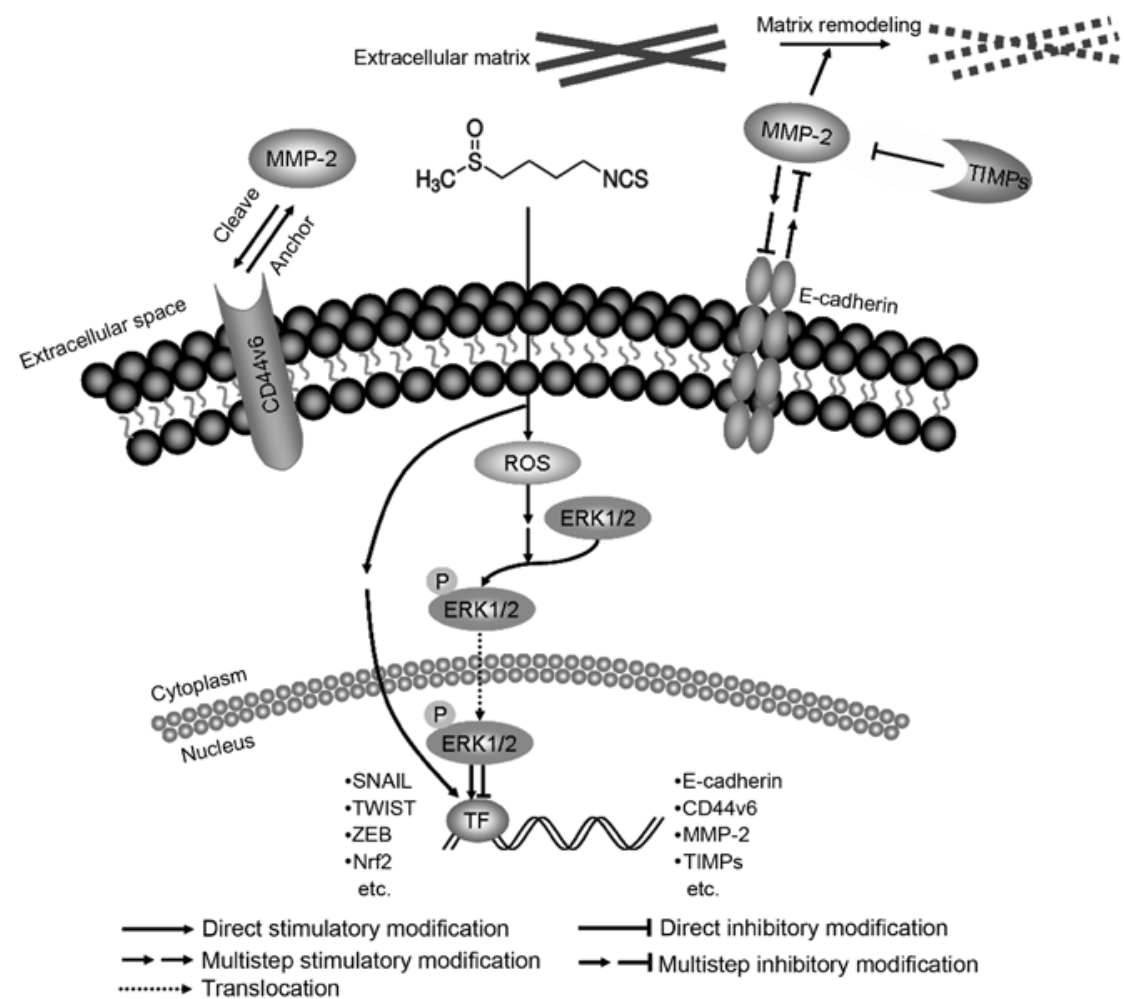

Figure 8. Assumed signaling map for SFN-triggered invasion inhibition. SFN, sulforaphane. 
the production of reactive oxygen species (ROS) by participation of SFN (46). SFN administration to prostate cancer cells led to ROS generation, which induced apoptosis and metastatic inhibition (14). In addition, we demonstrated that SFN upregulated E-cadherin and downregulated CD44v6 via the activation of ERK1/2. These two molecules are major players in mediating EMT. EMT is a reversible process, whereby the epithelial cells lose their junctions and polarity, reorganize their cytoskeleton, gain ability of motility, transit into mesenchymal cells and develop an invasive phenotype (30). The functional loss of E-cadherin is the typical characteristic of EMT and this contributes to the destabilization of adheren junctions, as well as tumor invasion and metastasis $(20,30,47,48)$. Therefore, SFN is capable of suppressing the process of EMT by inducing E-cadherin. EMT is manipulated by a group of transcription factors, including SNAIL, zinc-finger E-box-binding (ZEB) and TWIST. TWIST can be phosphorylated by ERK1/2, which promotes its nuclear import and functions, such as controlling the expression of E-cadherin (30). Notably, it has been suggested that MMPs regulate SNAIL and ZEB transcription, and modulate the E-cadherin level $(30,44)$. As a feedback loop, the loss of E-cadherin induced ZEB-dependent MMP-2 level in non-small cell lung cancer (32). Those findings are consistent with our results whereby SFN suppressed cell invasion by inducing E-cadherin and reducing MMP-2 in a dose-dependent manner. We also found that SFN significantly reduced the expression of CD44v6 and PD98059 attenuated the SFN-mediated reduction of CD44v6, suggesting that the downregulation of CD44v6 induced was through ERK1/2 activation. These results were coincident with those in the other studies. Overexpression of CD44v6 was found in primary prostate cancer tissues and metastases, but not in normal prostate tissues (3). It was also shown in the same study that knockdown of CD44v6 by siRNA decreased prostate cancer cell invasive and adhesive ability to hyaluronic acid (HA), suggesting that CD44v6 is an important participant in mediating tumor cell adhesion during metastasis (3). Another study showed that CD44 was a downstream effector of ERK1/2 inducing cell migration and invasion in vitro and tumor growth and metastasis in vivo in human oral cancer (19). It was reported that CD44v6 induced EMT in prostate cancer and the induction of EMT was accompanied by a shift in CD44 isoforms in breast cancer cells (49). Therefore, SFN was suggested to suppress EMT as well as invasion via the downregulation of CD44v6 and upregulation of E-cadherin.

A recent study described that the CD44v6 was found to be positively correlated with MMP-2 in papillary thyroid cancer (50). Furthermore, CD44 promoted invasion by anchoring MMPs on the tumor cell surface and it was cleaved by MMPs in the extracellular of several tumors (51). Emerging evidence indicates that MMPs can induce EMT during tumor development (39). MMP-2 is one of the most distinctive members of this family and facilitates prostate cancer cell invasion. Previous studies have demonstrated that MMP-2 expression is mediated by ERK1/2 (26,27,47,52). In the present study, we found that SFN significantly decreased MMP-2 expression and activity. However, the SFN-triggered inhibition of MMP-2 was not reversed by PD98059. Thus, the downregulation of MMP-2 expression and activity modulated by SFN was not via the ERK1/2 signaling pathway. Following the above observations, the question concerning how SFN regulated MMP-2 expression and activity is raised. One possible mechanism leading to decreased MMP-2 may be that SFN activates or inactivates some transcription factors, such as nuclear factor-кB (NF-кB), SNAIL and ZEB $(30,53)$. In addition, MMPs can also be regulated by their endogenous tissue inhibitors TIMPs, and the proteolytic activity of tumor cells depends on the balance between MMPs and TIMPs (47). Induction of TIMP-1/-2 by SFN has been proven in human bladder cancer cells (44). In this regard, we speculated that the upregulation of TIMP-1/-2 following the treatment of SFN may contribute to the inhibition of MMP-2 activity. More studies focusing on SFN-induced TIMP upregulation should therefore be conducted.

In conclusion, we have demonstrated that SFN suppressed invasion in human DU145 prostate cancer cells by modulating E-cadherin, CD44v6 and MMP-2, which linked to EMT (Fig. 8). Suppression of EMT was demonstrated to be an effective strategy against cancer metastasis $(30,47)$. The results suggested that $\mathrm{SFN}$ is a prospective drug to prevent invasion of prostate cancer cells.

\section{Acknowledgements}

The present study was supported by the National Natural Science Foundation of China (grant no. 81272843) and the Beijing Natural Science Foundation (grant no. 7132019). The funders had no role in the study design, data collection and analysis, decision to publish or preparation of the manuscript.

\section{References}

1. Siegel R, Naishadham D and Jemal A: Cancer statistics, 2013. CA Cancer J Clin 63: 11-30, 2013.

2. Chaturvedi S and Garcia JA: Novel agents in the management of castration resistant prostate cancer. J Carcinog 13: 5, 2014.

3. Ni J, Cozzi PJ, Hao JL, Beretov J, Chang L, Duan W, Shigdar S, Delprado WJ, Graham PH, Bucci J, et al: CD44 variant 6 is associated with prostate cancer metastasis and chemo-/radioresistance. Prostate 74: 602-617, 2014.

4. Nakano K, Komatsu K, Kubo T, Natsui S, Nukui A, Kurokawa S, Kobayashi M and Morita T: External validation of risk classification in patients with docetaxel-treated castration-resistant prostate cancer. BMC Urol 14: 31, 2014.

5. Cohen JH, Kristal AR and Stanford JL: Fruit and vegetable intakes and prostate cancer risk. J Natl Cancer Inst 92: 61-68, 2000.

6. Zhang SM, Hunter DJ, Rosner BA, Giovannucci EL, Colditz GA, Speizer FE and Willett WC: Intakes of fruits, vegetables, and related nutrients and the risk of non-Hodgkin's lymphoma among women. Cancer Epidemiol Biomarkers Prev 9: 477-485, 2000.

7. Ambrosone CB, McCann SE, Freudenheim JL, Marshall JR, Zhang Y and Shields PG: Breast cancer risk in premenopausal women is inversely associated with consumption of broccoli, a source of isothiocyanates, but is not modified by GST genotype. J Nutr 134: 1134-1138, 2004.

8. Dinkova-Kostova AT and Kostov RV: Glucosinolates and isothiocyanates in health and disease. Trends Mol Med 18: 337-347, 2012.

9. Choi S and Singh SV: Bax and Bak are required for apoptosis induction by sulforaphane, a cruciferous vegetable-derived cancer chemopreventive agent. Cancer Res 65: 2035-2043, 2005.

10. Talalay P and Fahey JW: Phytochemicals from cruciferous plants protect against cancer by modulating carcinogen metabolism. J Nutr 131 (Suppl): 3027S-3033S, 2001.

11. Conaway CC, Yang YM and Chung FL: Isothiocyanates as cancer chemopreventive agents: Their biological activities and metabolism in rodents and humans. Curr Drug Metab 3: 233-255, 2002. 
12. Lenzi M, Fimognari $C$ and Hrelia P: Sulforaphane as a promising molecule for fighting cancer. Cancer Treat Res 159: 207-223, 2014.

13. Cheung KL and Kong AN: Molecular targets of dietary phenethyl isothiocyanate and sulforaphane for cancer chemoprevention. AAPS J 12: 87-97, 2010.

14. Clarke JD, Dashwood RH and Ho E: Multi-targeted prevention of cancer by sulforaphane. Cancer Lett 269: 291-304, 2008.

15. Hecht SS: Inhibition of carcinogenesis by isothiocyanates. Drug Metab Rev 32: 395-411, 2000.

16. Li C, Zhou Y, Peng X, Du L, Tian H, Yang G, Niu J and Wu W: Sulforaphane inhibits invasion via activating ERK1/2 signaling in human glioblastoma U87MG and U373MG cells. PLoS One 9: e90520, 2014.

17. Deschênes-Simard X, Kottakis F, Meloche S and Ferbeyre G: ERKs in cancer: Friends or foes? Cancer Res 74: 412-419, 2014.

18. Zhou Y, Hu HY, Meng W, Jiang L, Zhang X, Sha JJ, Lu Z and Yao Y: MEK inhibitor effective against proliferation in breast cancer cell. Tumour Biol 35: 9269-9279, 2014.

19. Judd NP, Winkler AE, Murillo-Sauca O, Brotman JJ, Law JH, Lewis JS Jr, Dunn GP, Bui JD, Sunwoo JB and Uppaluri R: ERK1/2 regulation of CD44 modulates oral cancer aggressiveness. Cancer Res 72: 365-374, 2012.

20. Lau MT, So WK and Leung PC: Fibroblast growth factor 2 induces E-cadherin down-regulation via PI3K/Akt/mTOR and MAPK/ERK signaling in ovarian cancer cells. PLoS One 8: e59083, 2013.

21. Burotto M, Chiou VL, Lee JM and Kohn EC: The MAPK pathway across different malignancies: A new perspective. Cancer 120: 3446-3456, 2014.

22. Marshall CJ: Specificity of receptor tyrosine kinase signaling: Transient versus sustained extracellular signal-regulated kinase activation. Cell 80: 179-185, 1995.

23. Wu W, Ginsburg E, Vonderhaar BK and Walker AM: S179D prolactin increases vitamin D receptor and p21 through up-regulation of short $1 \mathrm{~b}$ prolactin receptor in human prostate cancer cells. Cancer Res 65: 7509-7515, 2005.

24. Liu Z, Yu X and Shaikh ZA: Rapid activation of ERK1/2 and AKT in human breast cancer cells by cadmium. Toxicol Appl Pharmacol 228: 286-294, 2008.

25. Yang TY, Chang GC, Chen KC, Hung HW, Hsu KH, Sheu GT and Hsu SL: Sustained activation of ERK and Cdk2/cyclin-A signaling pathway by pemetrexed leading to S-phase arrest and apoptosis in human non-small cell lung cancer A549 cells. Eur J Pharmacol 663: 17-26, 2011.

26. Li Z, Du L, Li C and Wu W: Human chorionic gonadotropin $\beta$ induces cell motility via ERK1/2 and MMP-2 activation in human glioblastoma U87MG cells. J Neurooncol 111: 237-244, 2013.

27. Li Z, Li C, Du L, Zhou Y and Wu W: Human chorionic gonadotropin $\beta$ induces migration and invasion via activating ERK1/2 and MMP-2 in human prostate cancer DU145 cells. PLoS One 8: e54592, 2013.

28. Wheelock MJ and Johnson KR: Cadherins as modulators of cellular phenotype. Annu Rev Cell Dev Biol 19: 207-235, 2003.

29. Cavallaro $U$ and Christofori G: Cell adhesion and signalling by cadherins and Ig-CAMs in cancer. Nat Rev Cancer 4: 118-132, 2004.

30. Lamouille S, Xu J and Derynck R: Molecular mechanisms of epithelial-mesenchymal transition. Nat Rev Mol Cell Biol 15: 178-196, 2014.

31. Li Q and Mattingly RR: Restoration of E-cadherin cell-cell junctions requires both expression of E-cadherin and suppression of ERK MAP kinase activation in Ras-transformed breast epithelial cells. Neoplasia 10: 1444-1458, 2008.

32. Bae GY, Choi SJ, Lee JS, Jo J, Lee J, Kim J and Cha HJ: Loss of E-cadherin activates EGFR-MEK/ERK signaling, which promotes invasion via the ZEB1/MMP2 axis in non-small cell lung cancer. Oncotarget 4: 2512-2522, 2013.

33. Nagano O, Okazaki S and Saya H: Redox regulation in stem-like cancer cells by CD44 variant isoforms. Oncogene 32: 5191-5198, 2013.

34. Khan SA, Cook AC, Kappil M, Günthert U, Chambers AF, Tuck AB and Denhardt DT: Enhanced cell surface CD44 variant (v6, v9) expression by osteopontin in breast cancer epithelial cells facilitates tumor cell migration: Novel post-transcriptional, post-translational regulation. Clin Exp Metastasis 22: 663-673, 2005.
35. Akisik E, Bavbek S and Dalay N: CD44 variant exons in leukemia and lymphoma. Pathol Oncol Res 8: 36-40, 2002.

36. Lee JL, Wang MJ, Sudhir PR, Chen GD, Chi CW and Chen JY: Osteopontin promotes integrin activation through outside-in and inside-out mechanisms: OPN-CD44V interaction enhances survival in gastrointestinal cancer cells. Cancer Res 67: 20892097, 2007.

37. Mulder JW, Wielenga VJ, Polak MM, van den Berg FM, Adolf GR, Herrlich P, Pals ST and Offerhaus GJ: Expression of mutant $\mathrm{p} 53$ protein and CD44 variant proteins in colorectal tumorigenesis. Gut 36: 76-80, 1995.

38. Jones JL and Walker RA: Control of matrix metalloproteinase activity in cancer. J Pathol 183: 377-379, 1997.

39. Orlichenko LS and Radisky DC: Matrix metalloproteinases stimulate epithelial-mesenchymal transition during tumor development. Clin Exp Metastasis 25: 593-600, 2008.

40. Bérubé M, Deschambeault A, Boucher M, Germain L, Petitclerc E and Guérin SL: MMP-2 expression in uveal melanoma: Differential activation status dictated by the cellular environment. Mol Vis 11: 1101-1111, 2005.

41. Sato T, Sakai T, Noguchi Y, Takita M, Hirakawa S and Ito A: Tumor-stromal cell contact promotes invasion of human uterine cervical carcinoma cells by augmenting the expression and activation of stromal matrix metalloproteinases. Gynecol Oncol 92: 47-56, 2004.

42. Di Nezza LA, Misajon A, Zhang J, Jobling T, Quinn MA, Ostör AG, Nie G, Lopata A and Salamonsen LA: Presence of active gelatinases in endometrial carcinoma and correlation of matrix metalloproteinase expression with increasing tumor grade and invasion. Cancer 94: 1466-1475, 2002.

43. Gupta P, Kim B, Kim SH and Srivastava SK: Molecular targets of isothiocyanates in cancer: Recent advances. Mol Nutr Food Res 58: 1685-1707, 2014.

44. Shan Y, Zhang L, Bao Y, Li B, He C, Gao M, Feng X, Xu W, Zhang $X$ and Wang S: Epithelial-mesenchymal transition, a novel target of sulforaphane via COX-2/MMP2, 9/Snail, ZEB1 and miR-200c/ZEB1 pathways in human bladder cancer cells. J Nutr Biochem 24: 1062-1069, 2013.

45. Hahm ER, Chandra-Kuntal K, Desai D, Amin S and Singh SV: Notch activation is dispensable for D, L-sulforaphane-mediated inhibition of human prostate cancer cell migration. PLoS One 7: e44957, 2012.

46. Jo C, Kim S, Cho SJ, Choi KJ, Yun SM, Koh YH, Johnson GV and Park SI: Sulforaphane induces autophagy through ERK activation in neuronal cells. FEBS Lett 588: 3081-3088, 2014.

47. Shen KH, Liao AC, Hung JH, Lee WJ, Hu KC, Lin PT, Liao RF and Chen PS: $\alpha$-Solanine inhibits invasion of human prostate cancer cell by suppressing epithelial-mesenchymal transition and MMPs expression. Molecules 19: 11896-11914, 2014.

48. Wu W and Walker AM: Human chorionic gonadotropin beta (HCGbeta) down-regulates E-cadherin and promotes human prostate carcinoma cell migration and invasion. Cancer 106: 68-78, 2006.

49. Brown RL, Reinke LM, Damerow MS, Perez D, Chodosh LA, Yang J and Cheng C: CD44 splice isoform switching in human and mouse epithelium is essential for epithelial-mesenchymal transition and breast cancer progression. J Clin Invest 121: 1064-1074, 2011.

51. Wu G, Zhou Y, Li T, Guo J and Zhou Z: Immunohistochemical levels of matrix metalloproteinase-2 and CD44 variant 6 protein in the diagnosis and lateral cervical lymph node metastasis of papillary thyroid carcinoma. J Int Med Res 41: 816-824, 2013.

52. Yu Q and Stamenkovic I: Localization of matrix metalloproteinase 9 to the cell surface provides a mechanism for CD44-mediated tumor invasion. Genes Dev 13: 35-48, 1999.

53. Chen Y, Zheng L, Liu J, Zhou Z, Cao X, Lv X and Chen F: Shikonin inhibits prostate cancer cells metastasis by reducing matrix metalloproteinase-2/-9 expression via AKT/mTOR and ROS/ERK1/2 pathways. Int Immunopharmacol 21: 447-455, 2014.

54. Wang Y, Li M, Xu Y, He N, Leng L and Li Z: Tumor necrosis factor- $\alpha$ regulates matrix metalloproteinase-2 expression and cell migration via ERK pathway in rat glomerular mesangial cells. Cell Biol Int 38: 1060-1068, 2014. 